\title{
Hadronic Correlators and Condensate Fluctuations in QCD Vacuum
}

\author{
Varun Sheel* ${ }^{*}$ Hiranmaya Mishra ${ }^{\dagger}$ and Jitendra C. Parikh ${ }^{\ddagger}$ \\ Theory Group, Physical Research Laboratory, Navrangpura, Ahmedabad 380 009, India
}

\begin{abstract}
Phenomenological results of equal time, point to point spatial correlation functions of hadronic currents are used to deduce the structure of the QCD vacuum. It is found that a model with only quark condensate is not adequate to explain the observations. Inclusion of condensate fluctuations (explicit four point structure in the vacuum) leads to excellent overall agreement with the phenomenological curves and parameters in various channels.

PACS number(s): 12.38.Gc
\end{abstract}

Typeset using REVTEX

*Electronic address: varun@prl.ernet.in

${ }^{\dagger}$ On leave from : The Mehta Research Institute for Mathematics and Mathematical Physics, 10 Kasturba Gandhi Marg, Allahabad - 211 022, India

${ }^{\ddagger}$ Electronic address: parikh@prl.ernet.in 
The most interesting question in Quantum Chromodynamics (QCD) is concerned with the nature of the vacuum state [1]. It is well known that the vacuum is non-trivial and is composed of gluon and quark field condensates [2,3]. Much of the understanding of nonperturbative phenomena in QCD is expected to result from a proper knowledge of the ground state of QCD.

In this note, we adopt a phenomenological approach to determine the salient features of the QCD vacuum. We consider here spacelike separated correlation functions of hadron currents in QCD vacuum [3]. More precisely, we use phenomenological results of equal time, point to point spatial ground state correlation functions of hadronic currents [4] to guide us towards a "true" structure of QCD vacuum.

As a first step, we employ the explicit construct for QCD vacuum with quark and gluon condensates proposed by us [5]. In this the trial ansatz for the QCD vacuum was [5]

$$
\left|v a c>=\exp \left(B_{F}^{\dagger}-B_{F}\right) \exp \left(B_{G}^{\dagger}-B_{G}\right)\right| 0>
$$

where $\mid 0>$ is the perturbative vacuum and $B_{F}^{\dagger}$ and $B_{G}^{\dagger}$ are the Bogoliubov operators corresponding to creation of quark antiquark pairs and gluon pairs respectively. Such a construct gives rise to the equal time propagator [6] as (with $x=|\vec{x}|$ ),

$$
\begin{aligned}
S_{\alpha \beta}(\vec{x}) & =\left\langle v a c\left|\frac{1}{2}\left[\psi_{\alpha}^{i}(\vec{x}), \bar{\psi}_{\beta}^{i}(0)\right]\right| v a c\right\rangle \\
& =\frac{1}{2} \frac{1}{(2 \pi)^{3}} \int e^{i \vec{k} \cdot \vec{x}} d \vec{k}[\sin 2 h(\vec{k})-(\vec{\gamma} \cdot \hat{k}) \cos 2 h(\vec{k})]
\end{aligned}
$$

where $h(\vec{k})$ describes the quark condensate [5]. Clearly $h(\vec{k}) \rightarrow 0$ gives the free massless quark propagator. In the limit of vanishing of constituent quark masses the model predicts absence of chiral symmetry breaking.

We next evaluate the correlation functions of hadronic currents in the vacuum defined by Eqs. (1)-(2). It turns out that without any approximation, the condensate structure uniquely determines the interacting quark propagator and the mesonic(baryonic) correlators are essentially squares(cubes) of the propagator. With a Gaussian ansatz for $h(\vec{k})$ [5], namely $\sin 2 h(\vec{k})=\exp \left(-R^{2} k^{2} / 2\right)$ we find that the behaviour of the quark propagator is similar to 
that obtained by Shuryak and Verbaarschott [7]. Further, the mesonic correlation functions were also qualitatively similar to phenomenological correlation functions in all channels except for the pseudoscalar(PS) channel. In this channel we did not get the strong attraction at intermediate ranges seen in the data. All these results depend very weakly on the choice of the functional form of $h(\vec{k})$.

In view of this outcome, it is obvious that some crucial physics is missing from our model, and hence the vacuum structure considered by us has to be supplemented by additional effects. In our framework, this means that the quark propagators alone do not determine the hadronic correlators. This implies that there ought to be explicit contribution arising from irreducible four point structure of the vacuum. Alternatively we can describe the irreducible four point vacuum structure as a manifestation of condensate fluctuations.

In order to proceed further with this idea of condensate fluctuations we suggest that the normal ordered operators with respect to the vacuum (Eq. (1)) do not annihilate the actual ground state of QCD which could be more complicated. In this case, we have a more general equation

$$
T \bar{\psi}_{\alpha}(\vec{x}) \psi_{\beta}(\vec{x}) \bar{\psi}_{\gamma}(0) \psi_{\delta}(0)=S_{\beta \gamma}(\vec{x}) S_{\delta \alpha}(-\vec{x})+: \bar{\psi}_{\alpha}(\vec{x}) \psi_{\beta}(\vec{x}) \bar{\psi}_{\gamma}(0) \psi_{\delta}(0):
$$

where the : denotes normal ordering with respect to the vacuum of Eq.(1). To include the effect of fluctuations we may write

$$
: \bar{\psi}_{\alpha}(\vec{x}) \psi_{\beta}(\vec{x}) \bar{\psi}_{\gamma}(0) \psi_{\delta}(0):=\Sigma_{\beta \gamma}(\vec{x}) \Sigma_{\delta \alpha}(-\vec{x})
$$

so that $\langle$ vac $|\Sigma \Sigma|$ vac $>=0$ but $\langle\Omega|\Sigma \Sigma| \Omega>\neq 0$ where $| \Omega>$ is the "new improved" QCD ground state including the condensate fluctuations.

With such a structure for the ground state of QCD the correlator takes the form

$$
R(\vec{x})=\operatorname{Tr}\left[S(\vec{x}) \Gamma^{\prime} S(-\vec{x}) \Gamma\right]+\operatorname{Tr}\left[\Sigma(\vec{x}) \Gamma^{\prime} \Sigma(-\vec{x}) \Gamma\right]
$$

with the extra term arising from the condensate fluctuations. The structure for the fluctuating field is arbitrary so far with all possible Dirac matrix structures (i.e. $1, \gamma_{5}, \gamma_{\mu}, \gamma_{\mu} \gamma_{5}$, $\left.\gamma_{\mu \nu} ; \mu \neq \nu\right)$ 
The experimental data demands that we choose the condensate fluctuation field such that it contributes maximally in the PS channel and should not affect the other channels very much. Such a condition restricts the choice for the fluctuating field to a structure of the type

$$
\Sigma_{\alpha \beta}(\vec{x})=\Sigma_{\alpha \beta}^{V}(\vec{x})+\Sigma_{\alpha \beta}^{S}(\vec{x}) \quad=\mu_{1}^{2}\left(\gamma^{i} \gamma^{j}\right)_{\alpha \beta} \epsilon_{i j k} \phi^{k}(\vec{x})+\mu_{2}^{2} \delta_{\alpha \beta} \phi(\vec{x})
$$

where the first term corresponds to vector fluctuations and the second to scalar. $\mu_{1}$ and $\mu_{2}$ in the above equations are dimensional parameters which give the strength of the fluctuations and $\phi^{k}(\vec{x})$ and $\phi(\vec{x})$ are vector and scalar fields such that

$$
<\Omega\left|\phi^{k}(\vec{x})\right| \Omega>=0 ; \quad<\Omega|\phi(\vec{x})| \Omega>=0
$$

and

$$
<\Omega\left|\phi^{i}(\vec{x}) \phi^{j}(0)\right| \Omega>=\delta^{i j} g_{V}(\vec{x}) ; \quad<\Omega|\phi(\vec{x}) \phi(0)| \Omega>=g_{S}(\vec{x})
$$

This implies an approximation that the ground state of QCD is also a condensate in the fluctuating fields. The functions $g_{S, V}(x)$ are at this stage arbitrary. It should be pointed out that the vector fluctuations contribute only to the PS and nucleon channels, while not contributing to the other physical channels. On the other hand the scalar fluctuations contribute to all channels but most to the vector and delta channels. It ought to be noted that to be consistent with phenomenology of correlators, the contribution of vector fluctuations should be greater than that of scalar fluctuations.

Since we do not expect fluctuations to be important for $\vec{x} \rightarrow 0$ and for large $x$, we want $g(\vec{x})$ to vanish in these two limits. With these properties in mind we take the function $g(\vec{x})$ as

$$
g_{V}(\vec{x})=\frac{1}{2 \pi^{2} x}\left[\mu_{1} K_{1}\left(\mu_{1} x\right)-\mu_{3} K_{1}\left(\mu_{3} x\right)\right] ; \quad g_{S}(\vec{x})=\frac{1}{2 \pi^{2} x}\left[\mu_{2} K_{1}\left(\mu_{4} x\right)-\mu_{5} K_{1}\left(\mu_{6} x\right)\right]
$$

so that the small $x$ behaviour of the correlator is the same as expected from the asymptotic freedom. $g_{V}(\vec{x})$ corresponds to taking the fluctuating field condensate function as difference 
of two propagators with different masses. Such a structure for the condensate function arises naturally e.g. in the $\phi^{4}$ field theory in the Gaussian effective potential calculations [8] where $\mu_{1}$ corresponds to the lagrangian mass parameter and $\mu_{3}$ corresponds to the variational parameter associated with the Gaussian ansatz. For $g_{S}(\vec{x})$ we had to consider a more general form (with extra parameters) because the contributions of the propagators and of the scalar fluctuations are comparable, requiring delicate cancellations.

The contributions of the propagator and the fluctuation fields to mesonic and baryonic channels are shown separately in cols. 3 and 4 of Table \. The parameters of the quark condensate function $h(\vec{k})$ and the of fluctuating fields $g_{S, V}$ are chosen so that our correlation functions are similar to those obtained from phenomenology. We have taken $R=0.60 \mathrm{fm}$ corresponding to $\langle\bar{\psi} \psi\rangle=-(304.45 \mathrm{MeV})^{3}$. This value is similar to the value $\langle\bar{\psi} \psi>=$ $-(307.4 \mathrm{MeV})^{3}$. considered in Ref. [9,10], though it is higher than the phenomenological value. Vector fluctuations dominate in the PS channel beyond $x=0.5 \mathrm{fm}$. This behaviour is reproduced by choosing $\mu_{1}=164.8$ and $\mu_{3}=69$. In the vector channel the propagator and fluctuation contributions are of the same order. If we choose $\mu_{2}=245, \mu_{4}=400, \mu_{5}=$ $1280.77, \mu_{6}=518$ we get reasonable agreement with phenomenological curves of correlators. The parameters $\mu_{1 \ldots 6}$ are in units of $\mathrm{MeV}$. The resulting correlators are plotted in Fig. 1.

We fit our results (solid curves of Fig. 11) to phenomenologically motivated forms for correlators parametrised in terms of mass, coupling and threshold of the corresponding particle [4]. We used the Marquardt method for non-linear least square fit. The method was very stable and the goodness of fit estimated from $\chi^{2}$ was reasonable for small and intermediate $x$, which is the region characterising the mass, coupling and threshold of a general correlator [4]. Our fitted parameters and those similarly obtained in lattice [4], instanton liquid model for QCD vacuum [9,10] and QCD sum rule [11] calculations are tabulated in Table $\mathbb{1 1}$.

As is evident from Fig. 1 and Table [I] our model of the vacuum gives results for the hadronic correlators that are comparable to those in the instanton model [9, 10] and lattice calculations [4]. The contribution of condensate fluctuations is most important and in some 
ways is related to the "hidden contribution" discussed by Shuryak [0,9].To summarise we have quite clearly shown that to be consistent with data, QCD vacuum must not only have quark condensates but must of necessity also have condensate fluctuations.

\section{ACKNOWLEDGMENTS}

VS and HM wish to acknowledge discussions with A. Mishra, and S. P. Misra. VS would like to thank Dr.(Mrs.) R. Suhasini for discussions on nonlinear curve fitting. HM thanks Theory Division of Physical Research Laboratory Ahmedabad for a visit and also acknowledges to the Council of Scientific and Industrial Research, Government of India for the research associateship 9/679(3)/95-EMR-I. 


\section{REFERENCES}

[1] R.P. Feynman, Nucl. Phys. B188 (1981) 479.

[2] E.V. Shuryak, The QCD vacuum, hadrons and the superdense matter, (World Scientific, Singapore, 1988).

[3] E.V. Shuryak, Rev. Mod. Phys. 65 (1993) 1.

[4] M.-C. Chu, J. M. Grandy, S. Huang and J. W. Negele, Phys. Rev. D48 (1993) 3340 ; ibid, Phys. Rev. D49 (1994) 6039 .

[5] A. Mishra, H. Mishra, S.P. Misra, P.K. Panda and Varun Sheel, Int. J. Mod. Phys. E5 (1996) 93.

[6] S. L. Adler and A.C. Davis, Nucl. Phys. B244 (1984) 469; R. Alkofer and P. A. Amundsen, Nucl. Phys. B306 (1988) 305 ; M. G. Mitchard, A. C. Davis and A. J. Macfarlane, Nucl. Phys. B325 (1989) 470 .

[7] E. V. Shuryak and J. J. M. Verbaarschot, Nucl. Phys. B410 (1993) 37.

[8] P.M. Stevenson, Phys. Rev. D32 (1985) 1389 ; H. Mishra and A.R. Panda, J. Phys. G (Part. and Nucl. Phys.)18 (1992) 1301.

[9] E. V. Shuryak and J. J. M. Verbaarschot, Nucl. Phys. B410 (1993) 55.

[10] T. Schäfer, E. V. Shuryak and J. J. M. Verbaarschot, Nucl. Phys. B412 (1994) 143.

[11] B.L. Ioffe, Nucl. Phys. B188 (1981) 317 ; V.B. Beleyaev and B.L. Ioffe, Zh. Eksp. Teor. Fiz. 83 (1982) 976 [Sov. Phys. JETP 56 (1982) 547 ] . 


\section{TABLES}

TABLE I. Meson currents and correlation functions

\begin{tabular}{lll}
\hline \hline CHANNEL CURRENT & CORRELATION FUNCTIONS $\left[\frac{R(x)}{R_{0}(x)}\right]$
\end{tabular}

Without fluctuations $^{\mathrm{a}} \quad$ Fluctuation contribution

$\left(\operatorname{Vector}\left(F^{V}\right)\right.$ and Scalar $\left.\left(F^{S}\right)\right)$

\begin{tabular}{|c|c|c|c|}
\hline Vector & $\bar{u} \gamma_{\mu} d$ & {$[F(x)]^{2}+\frac{\pi}{4} \frac{x^{6}}{R^{6}} e^{-x^{2} / R^{2}}$} & $\begin{array}{l}F^{V}=0 \\
F^{S}=8 \pi^{4} x^{6} g_{S}(2 x)\end{array}$ \\
\hline Pseudoscalar & $\bar{u} \gamma_{5} d$ & {$[F(x)]^{2}+\frac{\pi}{8} \frac{x^{6}}{R^{6}} e^{-x^{2} / R^{2}}$} & $\begin{array}{l}F^{V}=-48 \pi^{4} x^{6} g_{V}(2 x) \\
F^{S}=4 \pi^{4} x^{6} g_{S}(2 x)\end{array}$ \\
\hline Nucleon & $\epsilon_{a b c}\left[\tilde{u}^{a}(x) C \gamma_{\mu} u^{b}(x)\right] \gamma^{\mu} \gamma_{5} d^{c}(x)$ & {$[F(x)]^{3}+\frac{\pi}{16} \frac{x^{6}}{R^{6}} e^{-x^{2} / R^{2}} F(x)$} & $\begin{array}{l}F^{V}=-4 \pi^{4} x^{6} g_{V}(2 x) F(x \\
F^{S}=2 \pi^{4} x^{6} g_{S}(2 x) F(x)\end{array}$ \\
\hline Delta & $\epsilon_{a b c}\left[\tilde{u}^{a}(x) C \gamma_{\mu} u^{b}(x)\right] u^{c}(x)$ & {$[F(x)]^{3}+\frac{\pi}{4} \frac{x^{6}}{R^{6}} e^{-x^{2} / R^{2}} F(x)$} & $\begin{array}{l}F^{V}=0 \\
F^{S}=8 \pi^{4} x^{6} g_{S}(2 x) F(x)\end{array}$ \\
\hline
\end{tabular}

${ }^{\mathrm{a}} F(x)=\left[1+\frac{1}{2} x^{2} I(x)\right]$ where $I(x)=\int_{0}^{\infty}\left(\cos k x-\frac{\sin k x}{k x}\right) \frac{k e^{-R^{2} k^{2}}}{1+\left(1-e^{-R^{2} k^{2}}\right)^{1 / 2}} d k$ 
TABLE II. Fitted Parameters

\begin{tabular}{|c|c|c|c|c|}
\hline CHANNEL & SOURCE & $\mathrm{M}(\mathrm{GeV})$ & $\lambda$ & $\sqrt{s_{0}}(\mathrm{GeV})$ \\
\hline \multirow[t]{4}{*}{ Vector } & Ours & $0.78 \pm 0.005$ & $(0.42 \pm 0.041 \mathrm{GeV})^{2}$ & $2.07 \pm 0.02$ \\
\hline & Lattice & $0.72 \pm 0.06$ & $(0.41 \pm 0.02 \mathrm{GeV})^{2}$ & $1.62 \pm 0.23$ \\
\hline & Instanton & $0.95 \pm 0.10$ & $(0.39 \pm 0.02 \mathrm{GeV})^{2}$ & $1.50 \pm 0.10$ \\
\hline & Phenomenology & 0.78 & $(0.409 \pm 0.005 \mathrm{GeV})^{2}$ & $1.59 \pm 0.02$ \\
\hline \multirow[t]{4}{*}{ Pseudoscalar } & Ours & $0.137 \pm 0.0001$ & $(0.475 \pm 0.015 \mathrm{GeV})^{2}$ & $2.12 \pm 0.083$ \\
\hline & Lattice & $0.156 \pm 0.01$ & $(0.44 \pm 0.01 \mathrm{GeV})^{2}$ & $<1.0$ \\
\hline & Instanton & $0.142 \pm 0.014$ & $(0.51 \pm 0.02 \mathrm{GeV})^{2}$ & $1.36 \pm 0.10$ \\
\hline & Phenomenology & 0.138 & $(0.480 \mathrm{GeV})^{2}$ & $1.30 \pm 0.10$ \\
\hline \multirow[t]{5}{*}{ Nucleon } & Ours & $0.87 \pm 0.005$ & $(0.286 \pm 0.041 \mathrm{GeV})^{3}$ & $1.91 \pm 0.02$ \\
\hline & Lattice & $0.95 \pm 0.05$ & $(0.293 \pm 0.015 \mathrm{GeV})^{3}$ & $<1.4$ \\
\hline & Instanton & $0.96 \pm 0.03$ & $(0.317 \pm 0.004 \mathrm{GeV})^{3}$ & $1.92 \pm 0.05$ \\
\hline & Sum rule & $1.02 \pm 0.12$ & $(0.337 \pm 0.0 .014 \mathrm{GeV})^{3}$ & 1.5 \\
\hline & Phenomenology & 0.939 & $?$ & $1.44 \pm 0.04$ \\
\hline \multirow[t]{5}{*}{ Delta } & Ours & $1.52 \pm 0.003$ & $(0.341 \pm 0.041 \mathrm{GeV})^{3}$ & $3.10 \pm 0.008$ \\
\hline & Lattice & $1.43 \pm 0.08$ & $(0.326 \pm 0.020 \mathrm{GeV})^{3}$ & $3.21 \pm 0.34$ \\
\hline & Instanton & $1.44 \pm 0.07$ & $(0.321 \pm 0.016 \mathrm{GeV})^{3}$ & $1.96 \pm 0.10$ \\
\hline & Sum rule & $1.37 \pm 0.12$ & $(0.337 \pm 0.014 \mathrm{GeV})^{3}$ & 2.1 \\
\hline & Phenomenology & 1.232 & $?$ & $1.96 \pm 0.10$ \\
\hline
\end{tabular}




\section{FIGURES}
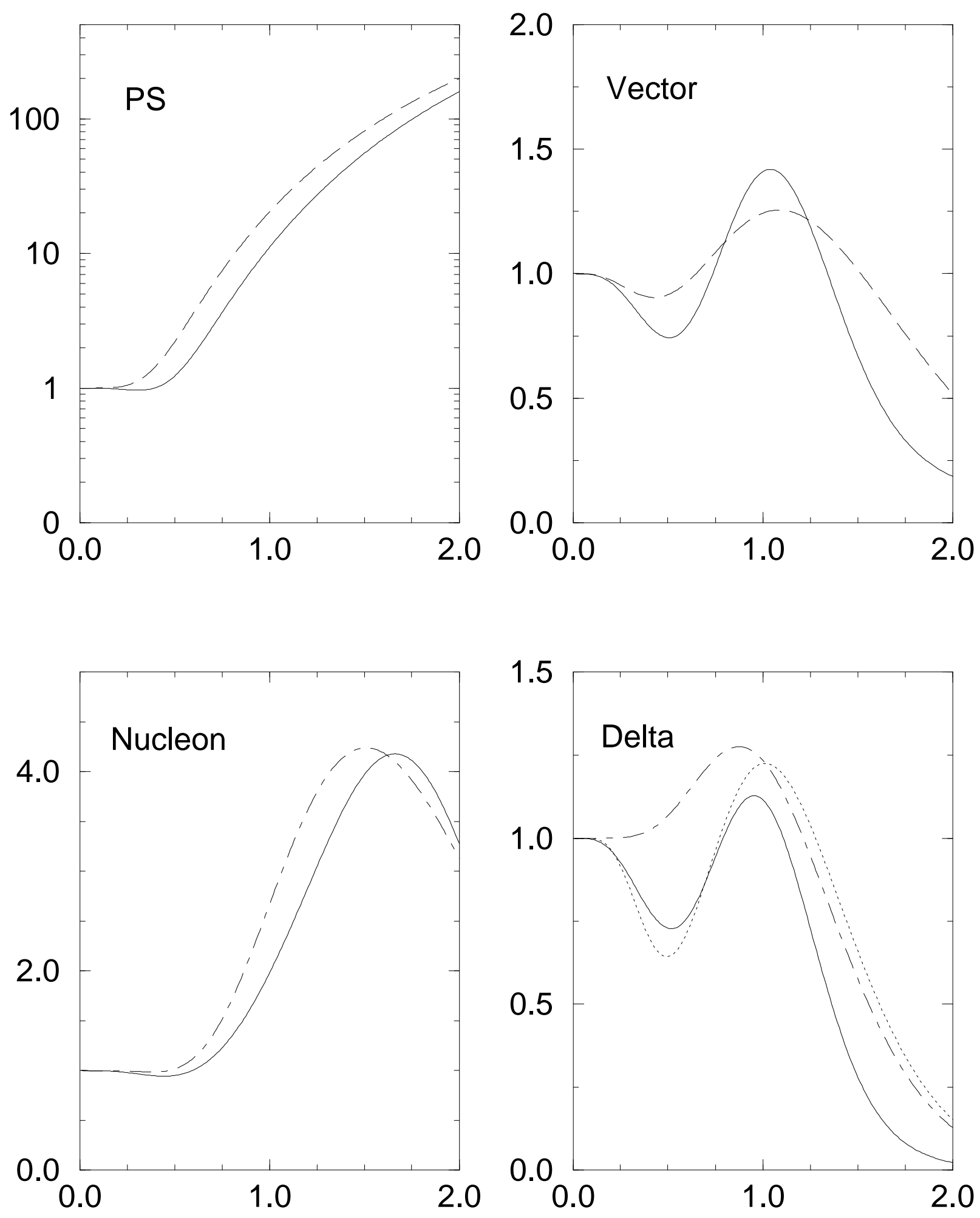
FIG. 1. The ratio of the hadron correlation functions in QCD vacuum to the correlation functions for noninteracting massless quarks $\frac{R(x)}{R_{0}(x)}$, vs. distance $\mathrm{x}$ (in fm). Our results are given by the solid curves. The empirical results determined by dispersion analysis of experimental data in Ref.[3] are shown by long dashed lines. The results from lattice calculations and instanton model are denoted by dotted and dot-dashed lines respectively. 